\title{
ASIGNACIÓN DE RECURSOS E INFLUENCIA DE LOS COTILEDONES EN EL CRECIMIENTO DE PLÁNTULAS DE NECTANDRA AMBIGENS (BLAKE) C.K.AlLEN (LAURACEAE) EN UNA SELVA TROPICAL HÚMEDA
}

\author{
Guadalupe Barajas-Guzmán y Javier Álvarez-SÁncheZ \\ Departamento de Ecología y Recursos Naturales, Facultad de Ciencias, Universidad Nacional Autónoma de \\ México; Circuito Exterior, Ciudad Universitaria; México 04510, D.F.; México. \\ 'Autor para la correspondencia. Tel 5622-4835. Fax 5622-4828, Correo-e: fjas@hp.fciencias.unam.mx
}

\begin{abstract}
Resumen: En la selva tropical húmeda, las especies cuyas plántulas tienen cotiledones, endospermo, o ambos, que funcionan como órganos de almacenamiento de reservas, tienen mayores probabilidades de permanecer en la regeneración de avanzada. En este estudio se analizó la influencia de los cotiledones en el crecimiento de plántulas de Nectandra ambigens, así como la asignación de biomasa hacia tallo, raíz y hojas. Una vez que germinaron, las semillas se transplantaron bajo el dosel de la selva. Se realizaron cinco cosechas y se registró el peso de los cotiledones y de las plántulas. El peso de las plántulas y los cotiledones fue significativamente diferente a través del tiempo. Se encontró una correlación negativa significativa entre la biomasa de los cotiledones y la de las plántulas, y un aumento en la biomasa fotosintética a expensas de la asignada a tallo. El trabajo demuestra la dependencia que tienen las plántulas de Nectandra ambigens de los recursos de los progenitores en los primeros 250 días, lo cual les confiere éxito para permanecer en el piso de la selva e integrarse a la regeneración de avanzada.

Palabras clave: cotiledones, crecimiento, Nectandra ambigens, plántulas, selva tropical húmeda.
\end{abstract}

\begin{abstract}
In the tropical rain forest, those species having cotyledons, endosperm, or both, which function as storage of parental resources, have a higher probability of surviving as part of the regeneration process. In this study, the influence of cotyledons on the growth of Nectandra ambigens seedlings was analyzed, as well as patterns of resource allocation to shoot, roots and leaves. After germination, seeds where grown under the forest canopy. Five surveys were made at two month intervals. The seedling and cotyledon biomass were significantly different along the time. A negative and significant correlation between cotyledon and seedling biomass was observed. An increase in photosynthetic biomass was measured getting resource from stem biomass and not from roots. This study shows the dependence of Nectandra ambigens seedlings on parental resources for the initial 250 days, which makes them successful in persisting on the forest floor and becoming part of the advanced regeneration.
\end{abstract}

Key words: cotyledons, growth, Nectandra ambigens, seedlings, tropical rain forest.

U no de los sistemas vegetales más diversos y complejos de la Tierra es la selva húmeda tropical. La diversidad de especies en este tipo de ecosistemas y su organización es el resultado de eventos que ocurren en los estadios de semilla, plántula y juvenil (Álvarez-Buylla y Martínez-Ramos, 1992), ya que son los estadios caracterizados por la mayor mortalidad de individuos (Brokaw, 1985; Martínez-Ramos y Soto-Castro, 1993). Para el mantenimiento de la diversidad, es necesaria la presencia de dichos estadios, de modo que nuevos individuos puedan reemplazar a los árboles adultos que van dejando lugares vacantes al morir
(Lieberman et al., 1985; Martínez-Ramos y ÁlvarezBuylla, 1995).

La calidad y la cantidad de semillas producidas por los individuos adultos son importantes, ya que estos atributos reflejan las estrategias evolutivas que se han desarrollado en estos ecosistemas. Por un lado, están las especies que producen un elevado número de semillas, de poco peso, con capacidad de dispersión a grandes distancias y que dan lugar a plántulas con cotiledones fotosintéticamente activos (Foster, 1986; Venable, 1988; Westoby et al., 1992); estas plántulas presentan una baja supervivencia en sitios con 
limitaciones de recursos. En cambio, existen otras especies que aumentan la calidad de sus semillas, asignando una mayor cantidad de reservas maternas al cotiledón o al endospermo de cada semilla (Smith y Fretwell, 1974), las cuales son de un peso elevado, de pobre dispersión y que dan lugar a plántulas con una supervivencia grande en ambientes desfavorables (Foster, 1986; Venable, 1988; Westoby et al., 1992).

Las semillas que pertenecen a este segundo grupo germinan bajo del dosel de la selva, dando lugar a plántulas que al estar en condiciones de umbría suprimen su crecimiento o crecen de manera muy lenta (Whitmore, 1978; Brokaw, 1985; Canham, 1985). De esta manera se integran a la comunidad denominada regeneración de avanzada (Martínez-Ramos, 1994), en la que dichas plántulas reemplazan a los árboles adultos de las especies persistentes (Martínez-Ramos y Soto-Castro, 1993). Este proceso está influido por la compleja dinámica de claros en la selva (Martínez-Ramos et al., 1988).

Diversos estudios han puesto de manifiesto que el componente mejor correlacionado con la masa de la semilla y con una supervivencia elevada de las plántulas en la sombra es el grosor de los cotiledones, expresado en términos de la asignación de biomasa por unidad de área (Kitajima, 1994; Saverimuttu y Westoby, 1996). Aún se desconoce cómo es que los cotiledones gruesos pueden dar ventajas en la supervivencia de las plántulas en la sombra. Además de que almacenan recursos maternos que son asignados directamente a la plántula (Foster, 1986; Oberbauer, 1990), los cotiledones gruesos tienen una menor tasa de ganancia de carbono por unidad de masa, disminuyendo por ello su metabolismo y aumentando la supervivencia de la plántula (Kitajima, 1996a). También se ha registrado que en condiciones de sombra la asignación de carbono para la defensa y el almacenaje resultan en una supervivencia elevada, a cambio de una menor cantidad de carbono disponible para el crecimiento inmediato (Bloom et al., 1985), de manera que el carbono asignado para crecimiento en las diferentes partes de la planta también marcará el éxito en el establecimiento y crecimiento (Kitajima y Fenner, 2000).

Es evidente que existen diversos factores, además de los recursos maternos, que influyen en el establecimiento y crecimiento de las plántulas, tales como la temperatura, la luz, las características del suelo, la cantidad de agua disponible para las plantas (Reynal y Bazzaz, 1973; Nobel, 1991) y la herbivoría (Janzen, 1976; Augspurger, 1984; Burdon, 1987). A pesar de esto, la mayoría de las investigaciones en las selvas húmedas se ha concentrado hasta ahora en las diferentes respuestas de las plantas a los gradientes de disponibilidad de luz (Vázquez-Yanes y OrozcoSegovia, 1990; Fenner, 1992; Dalling et al., 1999; Dalling et al., 2001; Osunkoya y Swanborough, 2001), y aunque es claro que la poca irradiación bajo el dosel de la selva impone limitaciones a la ganancia de carbono, es necesario estudiar la importancia de otros factores, entre ellos los recursos contenidos en los cotiledones que cubren al embrión en algunas semillas (Moussavi et al., 1997; Bungard et al., 2000; Coomes y Grubb, 2000).

En el caso de Nectandra ambigens (Blake) C.K.Allen, especie persistente de la selva cuyas plántulas crecen en condiciones de umbría en el sotobosque de la selva (Martínez-Ramos, 1994), los cotiledones parecen jugar un papel importante en su establecimiento. Además de que la germinación es hipógea, hay que añadir el hecho de que ésta es sincrónica y ello trae como resultado una fuerte competencia por el espacio, de manera que las reservas contenidas en los cotiledones se vuelven un factor determinante en el éxito de su establecimiento.

El objetivo de este estudio fue describir los cambios que ocurren en el peso de los cotiledones durante el crecimiento temprano de plántulas de Nectandra ambigens, bajo la hipótesis de que las plántulas hacen uso de las reservas maternas; por ello, se esperaría una correlación negativa entre el peso de los cotiledones y la biomasa de las plántulas. Además, se observaron los cambios en la asignación de biomasa a las diferentes partes de la plántula (tallo, raíz y hojas) a medida que crecían, esperando que los recursos se distribuyesen en su mayor parte en la elaboración de las láminas foliares y la raíz.

\section{Área de estudio}

El trabajo se realizó en la Estación de Biología Tropical "Los Tuxtlas", localizada en la porción sur del estado de Veracruz, en las coordenadas $95^{\circ} 04^{\prime}-95^{\circ} 09^{\prime} \mathrm{O}$ y $18^{\circ} 34^{\prime}$ - 18 36' N (Estrada et al., 1985). En la región se presenta un clima Af(m)w"(i')g (García, 1987; Soto y Gama, 1997), el cual es cálido húmedo, con una precipitación anual de $4,700 \mathrm{~mm}$ y una temperatura media anual de $24^{\circ} \mathrm{C}$. La mayor concentración de lluvia ocurre en el verano, a lo que se agrega la presencia de ciclones tropicales, si bien durante la época fría el área también es afectada por el desplazamiento de masas de aire frío y húmedo provenientes del norte (Soto y Gama, 1997). Los suelos en las partes altas de la Estación son andisoles, y alfisoles en las partes más bajas (Flores-Delgadillo et al., 1999).

El tipo de vegetación que se encuentra en el área natural de la Estación es una selva alta perennifolia (Miranda y Hernández-X., 1963). La selva presenta un dosel con alturas entre 30 y 35 m (Ibarra-Manríquez et al., 1997). Entre las especies más comunes se encuentran Astrocaryum mexicanum en el sotobosque, Pseudolmedia oxyphyllaria en el estrato medio y Nectandra ambigens domina en el dosel (Bongers et al., 1988); en la zona 50\% de las plantas son palmas (Ibarra-Manríquez et al., 1997).

Las semillas de Nectandra ambigens tienen viabilidad corta y germinación rápida ( $<3$ meses), y la humedad es el factor que la desencadena (Moreno, 1976); por ello, pueden 
germinar bajo la sombra de la selva formando una densa población de plántulas alrededor del árbol progenitor. El patrón espacial de esta especie es agregado (MartínezRamos y Álvarez-Buylla, 1995) debido a una dispersión reducida y a los bajos niveles (aproximadamente 50\%) de depredación que tienen las semillas (Córdova, 1979).

La infrutescencia de Nectandra ambigens tiene un tamaño de 10 a 25 cm de largo; el fruto es drupáceo, de 25 a $35 \mathrm{~mm}$ de largo y de 23 a $26 \mathrm{~mm}$ de ancho, y contiene una semilla por fruto; el endocarpo mide de 18 a $28 \mathrm{~mm}$ de largo y de 16 a $20 \mathrm{~mm}$ de ancho, y las semillas poseen cotiledones morado-rosados. La plántula es criptocotilar, con germinación hipógea; sus hojas son alternas y simples, y tienen el margen entero (Ibarra-Manríquez, 1985).

\section{Materiales y métodos}

Se recolectaron 150 semillas de Nectandra ambigens. A finales de enero de 1990 éstas se colocaron para su germinación en la sombra bajo el dosel de la selva en charolas con tierra tamizada; fueron regadas mientras germinaron. De las 150 germinaron 100; de ellas se eligieron 56 al azar y se transplantaron en abril de 1990, con dos semanas de edad (cuadro 1), a bolsas de plástico negro con suelo tamizado y homogeneizado en cantidad aproximada de 2 $\mathrm{kg}$ por bolsa. Al momento del trasplante, las plántulas de $N$. ambigens presentaban una raíz bien desarrollada, un tallo flexible y fuerte, y los cotiledones se encontraban moderadamente plegados.

Cuadro 1. Edad de las plántulas de Nectandra ambigens, tiempo transcurrido desde el transplante, biomasa total (raíz + tallo + hojas) en peso seco de las plántulas ( \pm e.e.) y tasa relativa de crecimiento para cada una de las cosechas.

\begin{tabular}{cccc}
$\begin{array}{c}\text { Edad de las } \\
\text { plántulas } \\
\text { (días) }\end{array}$ & $\begin{array}{c}\text { Tiempo a partir } \\
\text { del transplante } \\
\text { (días) }\end{array}$ & $\begin{array}{c}\text { Biomasa total } \\
\text { de las } \\
\text { plántulas }(g)\end{array}$ & $\begin{array}{c}\text { Tasa relativa } \\
\text { de crecimiento } \\
\left(\mathrm{g} \mathrm{g}^{-1} \text { día }\right.\end{array}$ \\
\hline 14 & 0 (abril) & $0.141 \pm 0.005$ & \\
61 & 47 (mayo) & $0.308 \pm 0.007$ & $0.0166 \pm 0.0008$ \\
104 & 90 (junio) & $0.503 \pm 0.006$ & $0.0114 \pm 0.0006$ \\
183 & 169 (septiembre) & $0.513 \pm 0.006$ & $0.0003 \pm 0.0003$ \\
253 & 239 (noviembre) & $0.639 \pm 0.006$ & $0.0031 \pm 0.0002$
\end{tabular}

Dentro de la selva se aisló un área con malla de mosquitero blanca con una superficie de $16 \mathrm{~m}^{2}$ y una altura de $2.5 \mathrm{~m}$; su función fue la de mantener a las plántulas en un ambiente lo más similar posible a las condiciones naturales, así como de protegerlas de la herbivoría, de la caída de hojarasca y de la lluvia de semillas. Las plántulas se fueron rotando periódicamente para eliminar efectos causados por la posición y fueron regadas hasta el inicio de la estación de lluvias a finales de mayo; posteriormente la precipitación fue suficiente para que se mantuviesen en buen estado. A lo largo del experimento, de abril a noviembre de 1990, se realizaron cinco cosechas a intervalos de dos meses aproximadamente, de ocho réplicas por muestreo. Las muestras se secaron a $90^{\circ} \mathrm{C}$ durante $48 \mathrm{~h}$.

Análisis de datos. El crecimiento de las plántulas se evaluó a través del peso seco (g) de la biomasa total (raíces + tallo + hojas). Con el fin de evaluar las diferencias a través del tiempo para los datos de biomasa de las plántulas y de los cotiledones, se realizó un análisis de varianza (ANOVA) de una vía (Zar, 1999). Cuando el análisis indicó diferencias significativas, se aplicó la prueba de Tukey (Zar, 1999) para determinar qué tiempos fueron diferentes entre sí.

Se obtuvo el coeficiente de correlación de Pearson entre la biomasa total (g) de las plántulas y la biomasa (g) de los cotiledones (Sokal y Rohlf, 1979). Se calculó también la tasa relativa de crecimiento promedio (Hunt, 1978):

$$
\mathrm{R}=(\ln \mathrm{Wf}-\ln \mathrm{Wi}) /(\mathrm{Tf}-\mathrm{Ti})
$$

donde: $\mathrm{R}=$ tasa relativa de crecimiento $\left(\mathrm{g} \mathrm{g}^{-1}\right.$ día $\left.^{-1}\right), \mathrm{Wf}=$ peso final, $\mathrm{Wi}=$ peso inicial, $\mathrm{Tf}=$ tiempo final, $\mathrm{Ti}=$ tiempo inicial, $\ln =$ logaritmo natural.

Para analizar la asignación de biomasa de las plántulas, se consideraron las proporciones de biomasa de tallo, raíz y hojas con respecto al peso total de la plántula (PBT, PBR y $\mathrm{PBH}$, respectivamente).

\section{Resultados}

Se encontraron diferencias significativas en el peso de las plántulas de Nectandra ambigens entre las cinco cosechas realizadas a lo largo del experimento $\left(F_{4,35}=1072.31 ; P<\right.$ 0.0001). El peso fue estadísticamente diferente entre los cinco periodos registrados, a excepción de los correspondientes a las cosechas efectuadas a los 90 y 170 días $(0.50 \pm 0.006$, $0.51 \pm 0.006 \mathrm{~g}$, respectivamente). Los valores más altos se alcanzaron a los 240 días $(0.64 \pm 0.005 \mathrm{~g})$ (figura 1$)$.

El peso de los cotiledones también presentó diferencias significativas a través del tiempo $\left(F_{4,35}=508.07 ; P<\right.$ $0.0001)$, a excepción nuevamente de los pesos registrados a los 90 y 170 días $(0.36 \pm 0.008,0.33 \pm 0.008$ g, respectivamente), para los que no se registraron diferencias significativas. Los valores más altos se observaron al inicio del experimento $(1.06 \pm 0.033 \mathrm{~g})$, y los más bajos al final del mismo $(0.107 \pm 0.007 \mathrm{~g})$ (figura 1$)$. Se encontró una correlación negativa $(r=-0.971)$ significativa $\left(F_{1,38}=\right.$ 627.99; $P<0.00001$ ) entre la biomasa de los cotiledones y la biomasa de las plántulas (figura 1).

Por lo que respecta a la tasa relativa de crecimiento, las plántulas presentaron su valor máximo en la segunda 


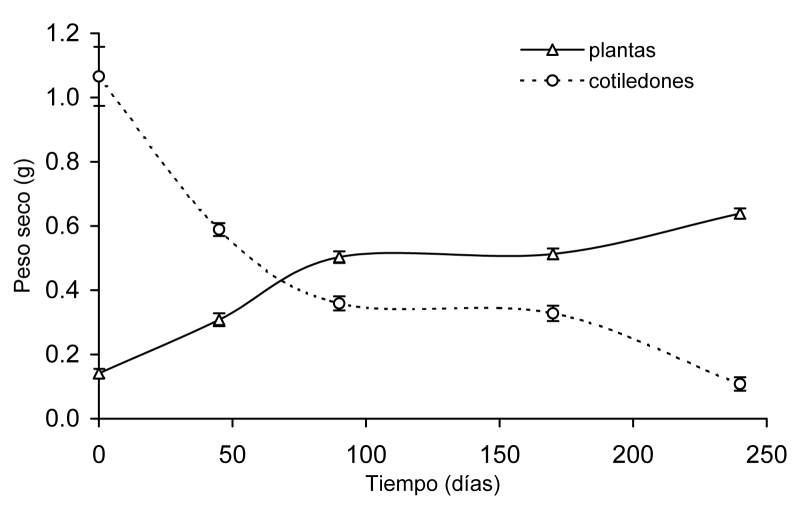

Figura 1. Pérdida y ganancia de peso seco (g) en los cotiledones y en plántulas de Nectandra ambigens a lo largo de 240 días (media + $2 \mathrm{EE}$ ).

cosecha (0.0166 g g ${ }^{-1}$ día $\left.^{-1} \pm 0.0008\right)$, y a partir de la tercera cosecha los incrementos disminuyeron (cuadro 1), aunque a partir de los 160 días, se observó nuevamente un cambio de biomasa en los cotiledones y las plántulas (figura 1), lo cual coincidió con un pequeño incremento en la tasa relativa de crecimiento de las plántulas (cuadro 1).

Por lo que respecta a la asignación de biomasa (figura 2), en el tiempo cero (que corresponde al transplante), cuando las plántulas contaban con dos semanas de edad (cuadro 1), 75\% de la asignación de la biomasa correspondió al tallo y el $25 \%$ restante a la raíz; en este período todavía no había tejido fotosintético. A los 45 días ocurrió un cam-

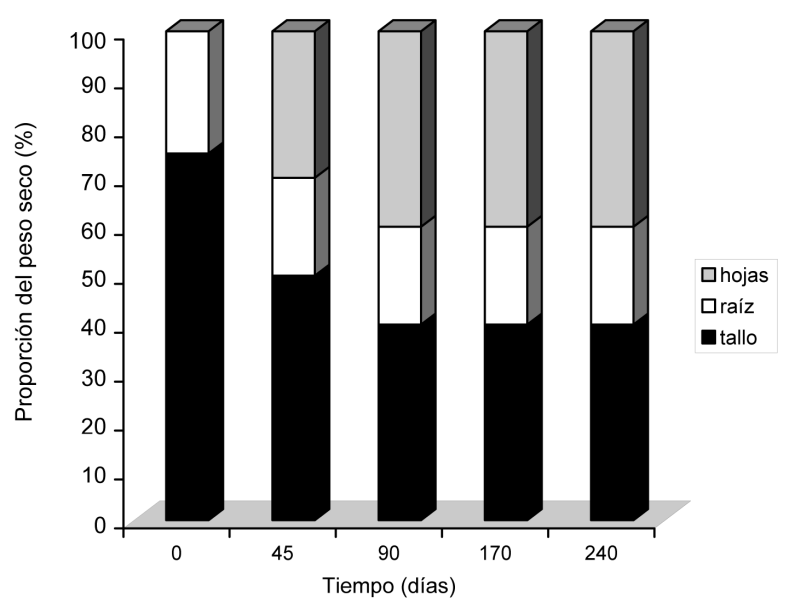

Figura 2. Cambios en el porcentaje de peso seco para la asignación de recursos de la planta. Se muestran las diferentes estructuras: raíz, tallo y hojas, a lo largo de 240 días. bio importante, ya que se asignó biomasa tanto a raíz y tallo como a las hojas, lo que originó una reducción en la biomasa asignada particularmente a tallo (ahora sólo de 50\%), aumentando considerablemente la asignación hacia las hojas (30\%). En ese momento se observó una reducción de biomasa de $5 \%$ a la raíz. A los 90 días volvieron a darse cambios, ya que la asignación a tallo se redujo en un $10 \%$, mismo que ganó el tejido fotosintético, mientras que la asignación a raíz no tuvo cambios; a partir de este momento los porcentajes se mantuvieron constantes hasta el término del estudio.

\section{Discusión}

Diversos estudios han puesto de manifiesto que el tamaño de las semillas está positivamente correlacionado con la tolerancia que tienen las plántulas a varios factores abióticos estresantes, entre ellos la falta de luz (Foster, 1986; Westoby et al., 1992; Kitajima y Fenner, 2000; Paz y Martínez-Ramos, 2003). Nectandra ambigens, por poseer una semilla grande y con reservas maternas, es una especie tolerante a la sombra; a pesar de que las plántulas se cosecharon cada dos meses aproximadamente, no se presentó mortalidad natural durante el periodo de la investigación, aunque evidentemente influyó el hecho de que estuvieran creciendo en condiciones de aislamiento dentro de la selva que evitaron que, por ejemplo, fueran dañadas por herbívoros.

Respecto a la tasa relativa de crecimiento (TRC), ésta resultó ser bastante baja en comparación con las tasas reportadas para otras especies tolerantes en el trabajo de Kitajima (1996b): la tasa promedio más alta de Nectandra ambigens fue inferior a la de Amphipterigium adstringens (Julianaceae) ( $\left.0.111 \mathrm{~g} \mathrm{~g}^{-1} \mathrm{dí}^{-1}\right)$, mientras que la tasa promedio más baja tuvo valores menores que la de Poulsenia armata (Moraceae) (0.008 $\mathrm{g} \mathrm{g}^{-1}$ día $\left.^{-1}\right)$.

Se ha reportado que las plántulas de semillas grandes en condiciones de umbría disminuyen su tasa de crecimiento. Foster (1986) sugiere que esto se debe a que las semillas grandes podrían proporcionar suficientes reservas a las plántulas, para producir compuestos secundarios para la defensa y poder persistir en el piso de la selva, o bien suficiente energía para reemplazar los tejidos dañados o perdidos, de manera que el carbono asignado al crecimiento disminuye y con ello la TRC. Apoyando esta idea, Armstrong y Westoby (1993) encontraron que las plántulas de semillas grandes fueron más tolerantes al daño. Por otra parte, Bonfil (1998) demostró que las plántulas derivadas de semillas grandes toleraron mejor la pérdida de cotiledones al enfrentar mejor el estrés ambiental. Se ha observado que este tipo de plántulas pueden sobrevivir en estas condiciones, mientras no sean atacadas por microorganismos patógenos. Otros estudios han demostrado que la mortalidad de las plántulas en condiciones de umbría es causada 
por hongos (Augspurger, 1984; Martínez-Ramos, 1991).

Augspurger (1984) señala que una vez que las plántulas han usado las reservas de la semilla en condiciones de umbría, disminuyen su tasa de crecimiento o la anulan, lo cual indica que están muy cerca de su punto de compensación de luz. Esta autora encontró evidencia para plántulas de 18 especies neotropicales de que la baja tasa relativa de crecimiento y la alta supervivencia en condiciones de umbría estuvieron correlacionadas con la densidad de la madera de los adultos y no con el peso de las reservas de la semilla. Son necesarios más estudios al respecto para dilucidar las hipótesis correctas.

En cuanto a la asignación de recursos, Nectandra ambigens, después de germinar, ganó altura y longitud de raíz, y posteriormente asignó recursos principalmente a las hojas (figura 2), presentándose una reducción importante en la proporción de peso del tallo. Esto se puede interpretar en el sentido de la importancia de extender el área foliar para captar la poca luz que penetra hasta el piso de la selva, pero no a expensas de la raíz, ya que es la responsable de la absorción de nutrimentos y de agua. Sin embargo, Lewis y Tanner (2000) estudiaron la asignación de biomasa en un par de especies de plántulas en un bosque tropical, una demandante de luz y la otra tolerante a la sombra, y encontraron que las dos especies incrementaron la asignación a las hojas a expensas de la biomasa asignada a la raíz. Por otro lado, plántulas de Cordia megalantha y Lonchocarpus guatemalensis (también tolerantes a la sombra de acuerdo con Martínez-Ramos, 1985) crecieron durante un año en un sitio abierto de la región de Los Tuxtlas, y en ese periodo se incrementó la biomasa asignada a raíz a expensas de la de hojas, mientras que la biomasa de tallo se mantuvo constante (Bongers et al., 1988).

La asignación de recursos de las plántulas hacia las diferentes partes que las forman (raíz, tallo y hojas) tiene que ver con que, en condiciones ambientales particulares y con la plasticidad de la especie, involucra el compromiso que tienen para crecer, para la defensa contra herbívoros y plagas, para reparar los tejidos dañados y para almacenaje (Kitajima y Fenner, 2000). A este respecto, MartínezRamos (1991) reporta que en zonas dominadas por Nectandra ambigens en la selva de Los Tuxtlas, en donde la densidad de plántulas fue de tres individuos por $\mathrm{m}^{2}$, las plántulas mostraron poco daño causado por vertebrados. Sin embargo, se ha encontrado evidencia de que éstas sufren daño causado por patógenos y por insectos (GarcíaGuzmán, 1990); además, si se presenta adición de hojarasca, disminuye la tasa relativa de crecimiento (GarcíaGuzmán y Benítez-Malvido, 2003). Estos factores disminuyen drásticamente la densidad de las plántulas en al menos tres años (Martínez-Ramos, 1991). Durante este tiempo las plántulas tienen alguna oportunidad de formar parte de la regeneración de avanzada, siempre y cuando aseguren primero su establecimiento.
En conclusión, este trabajo muestra evidencia del grado en que las plántulas de Nectandra ambigens dependen de los recursos maternos, al menos durante los primeros 250 días de su desarrollo, y que la expansión del tejido fotosintético en esta especie ocurrió a expensas de la biomasa asignada al tallo.

\section{Agradecimientos}

Los recursos para la realización de este trabajo fueron obtenidos gracias al apoyo del Proyecto DGAPA-UNAM IN-218096. Los autores agradecen la colaboración en el trabajo de campo a Irene Sánchez-Gallén, Patricia Guadarrama y Yukiko Sakurai.

\section{Literatura citada}

Álvarez-Buylla E. y Martínez-Ramos M. 1992. The demography of a neotropical pioneer tree: an evaluation of the pioneer-climax paradigm. Journal of Ecology 80:275-290.

Armstrong D. y Westoby M. 1993. Seedlings from large seeds tolerate defoliation better: a test using phylogenetically independent contrasts. Ecology 74:1092-1116.

Augspurger C. 1984. Light requirements of neotropical tree seedlings: a comparative study of growth and survival. Journal of Ecology 72:777-795.

Bloom A., Chapin F. y Mooney H. 1985. Resource limitation in plants: an economic analogy. Annual Review of Ecology and Systematics 16:363-392.

Bonfil C. 1998. The effects of seed size, cotyledon reserves, and herbivory on seedling survival and growth in Quercus rugosa and Q. laurina (Fagaceae). American Journal of Botany 85:7987.

Bongers F., Popma J. e Iriarte-Vivar S. 1988. Response of Cordia megalantha Blake seedlings to gap environments in tropical rain forest. Functional Ecology 2:379-390.

Bongers F., Pompa J., Meave del Castillo J. y Carabias J. 1988. The structure and floristic composition of the lowland rain forest of Los Tuxtlas, Mexico. Vegetatio 74:55-80.

Brokaw N. 1985. Gap phase regeneration in a tropical forest. Ecology 66:682-687.

Bungard R., Press M. y Scholes J. 2000. The influence of nitrogen on rain forest dipterocarp seedlings exposed to a large increase in irradiance. Plant Cell and Environment 23:11831194.

Burdon J. 1987. Diseases and Plant Population Biology. Cambridge University Press, Cambridge.

Canham C. 1985. Suppression and release during canopy recruitment in Acer saccharum. Bulletin of the Torrey Botanical Club 112:134-145.

Coomes A. y Grubb J. 2000. Impacts of root competition in forests and woodlands: A theoretical framework and review of experiments. Ecological Monographs 70:171-207.

Córdova M. 1979. Efectos de la densidad, la distancia al árbol progenitor y la depredación en el crecimiento y sobrevivencia de plántulas de Nectandra ambigens (Blake) C.K.Allen. Tesis de licenciatura, Facultad de Ciencias, Universidad Nacional 
Autónoma de México, México D.F., 132 pp.

Dalling J., Lovelock C. y Hubbell S. 1999. Growth responses of seedlings of two neotropical pioneer species to simulated forest gap environments. Journal of Tropical Ecology 15:827839.

Dalling J., Winter K., Nason J., Hubbell S., Murawski D. y Hamrick J. 2001. The unusual life history of Alseis blackiana: A shade-persistent pioneer tree? Ecology 82:933-945.

Estrada A., Coates-Estrada R. y Martínez-Ramos M. 1985. La Estación de Biología Tropical Los Tuxtlas: un recurso para el estudio y conservación de las selvas del trópico húmedo. En: Gómez-Pompa A. y Del Amo S. Eds. Investigaciones sobre la Regeneración de Selvas Altas en Veracruz, México, pp. 379393, Vol. II, INIREB y Alhambra, México, D.F.

Fenner M. 1992. Seed Ecology. Chapman \& Hall, Nueva York.

Flores-Delgadillo L., Sommer-Cervantes I., Alcalá-Martínez J. y Álvarez-Sánchez J. 1999. Estudio morfogenético de algunos suelos de la región de Los Tuxtlas, Veracruz, México. Revista Mexicana de Ciencias Geológicas 16:81-88.

Foster S. 1986. On the adaptative value of large seed for tropical moist forest tree: a review and synthesis. Botanical Review 52:269-299.

García E. 1987. Modificaciones al Sistema de Clasificación Climática de Köppen. Editado por la autora, México, D.F.

García-Guzmán G. 1990. Estudio sobre la ecología de patógenos en el follaje de plantas en la selva de Los Tuxtlas. Tesis de maestría, Facultad de Ciencias, Universidad Nacional Autónoma de México, México, D.F., 150 pp.

García-Guzmán G. y Benítez-Malvido J. 2003. Effect of litter on the incident of leaf-fungal pathogens and herbivory in seedlings of the tropical tree Nectandra ambigens. Journal of Tropical Ecology 19:171-177.

Hunt R. 1978. Plant Growth Analysis. Arnold, Londres.

Ibarra-Manríquez G. 1985. Estudios preliminares sobre la flora leñosa de la Estación de Biología Tropical Los Tuxtlas, Veracruz, México. Tesis de licenciatura, Facultad de Ciencias, Universidad Nacional Autónoma de México, México, D.F., $264 \mathrm{pp}$.

Ibarra-Manríquez G., Martínez-Ramos M., Dirzo R. y NúñezFarfán J. 1997. La vegetación. En: González-Soriano E., Dirzo R. y Vogt R. Eds. Historia Natural de Los Tuxtlas, pp. 61-85, Universidad Nacional Autónoma de México, México, D.F.

Janzen D. 1976. Reduction of Macuna andreana (Leguminosae) seeding fitness by artificial seed damage. Ecology 57:826-828.

Kitajima K. 1994. Relative importance of photosynthetic traits and allocation patterns as correlates of seedling shade tolerance of 13 tropical trees. Oecologia 98:419-428.

Kitajima K. 1996a. Cotyledon functional morphology, seed reserve utilization, and regeneration niches of tropical tree seedlings. En: Swaine M. Ed. The Ecology of Tropical Forest Tree Seedlings, pp. 193-208, UNESCO, París.

Kitajima K. 1996b. Ecophysiology of tropical tree seedlings. En: Mulkey S., Chazdon R. y Smith A. Eds. Tropical Forest Plant Ecophysiology, pp. 559-596, Chapman \& Hall, Nueva York.

Kitajima K. y Fenner M. 2000. Ecology of seedling regeneration. En: Fenner M. Ed. Seeds: The Ecology of Regeneration in Plant Communities, pp. 331-359, 2a ed., CAB Publishing, Londres.
Lewis S. y Tanner E. 2000. Effects of above and belowground competition on growth and survival of rain forest tree seedlings. Ecology 81:2525-2538.

Lieberman D., Lieberman M., Peralta R. y Hartshorn G. 1985. Mortality patterns and stand turnover rates in a wet tropical forest in Costa Rica. Journal of Ecology 73:915-926.

Martínez-Ramos M. 1985. Claros, ciclos vitales de los árboles tropicales y regeneración natural de las selvas altas perennifolias. En: Gómez-Pompa A. y Del Amo S. Eds. Investigaciones sobre la Regeneración de Selvas Altas en Veracruz, México, pp. 191-239, Vol. II, INIREB, Alhambra, México, D.F.

Martínez-Ramos M.1991. Patrones y mecanismos en la comunidad de plántulas de una selva húmeda neotropical. Tesis doctoral, UACPyP-CCH, Universidad Nacional Autónoma de México, México, D.F., 159 pp.

Martínez-Ramos M. 1994. Regeneración natural y diversidad de especies arbóreas en selvas húmedas. Boletín de la Sociedad Botánica de México 54:179-24.

Martínez-Ramos M. y Álvarez-Buylla E. 1995. Ecología de poblaciones de plantas en una selva húmeda de México. Boletín de la Sociedad Botánica de México 56:121-153.

Martínez-Ramos M., Álvarez-Buylla E., Sarukhán J. y Piñero D. 1988. Treefall age determination and gap dynamics in a tropical forest. Journal of Ecology 76:700-716.

Martínez-Ramos M. y Soto-Castro A. 1993. Seed rain and advanced regeneration in a tropical rain forest. Vegetatio 108:299-318.

Miranda F. y Hernández-X. E. 1963. Los tipos de vegetación de México y su descripción. Boletín de la Sociedad Botánica de México 28:29-178.

Moreno P. 1976. Latencia y viabilidad de semillas de vegetación primaria. En: Gómez-Pompa A., Vázquez-Yanez C., Del Amo S. y Butanda A. Eds. Investigaciones sobre la Regeneración de Selvas Altas en Veracruz, México, pp. 527-545, Vol. I, INIREB, CECSA, México, D.F.

Moussavi N., Rengel Z., Pearson J. y Hollamby G. 1997. Dynamics of nutrient remobilisation from seed of wheat genotypes during imbibition, germination and early seedling growth. Plant and Soil 197:271-280.

Nobel P. 1991. Physicochemical and Environmental Plant Physiology. Academic Press, San Diego.

Osunkoya O. y Swanborough W. 2001. Reproductive and ecophysiological attributes of the rare Gardenia actinocarpa (Rubiaceae) compared with its common co-occurring congener, G. ovularis. Australian Journal of Botany 49:471-478.

Oberbauer F. 1990. Seed weight and rooting depth of seedlings of Costa Rica wet forest trees. Revista de Biología Tropical 38:475-478.

Paz H. y Martínez-Ramos M. 2003. Seed mass and seedling performance within eight species of Psychotria (Rubiaceae). Ecology 84:439-450.

Reynal J. y Bazzaz F. 1973. Establishment of early successional plant populations on forest and prairie soil. Ecology 54:13351341.

Saverimuttu T. y Westoby M. 1996. Seedling longevity under deep shade in relation to seed size. Journal of Ecology 84:681689.

Smith C. y Fretwell S. 1974. The optimal balance between size 
and numer of offspring. American Naturalist 108:499-506.

Sokal R. y Rohlf J. 1979. Biometría: Principios y Métodos Estadísticos en la Investigación Biológica. Blume, Madrid.

Soto M. y Gama L. 1997. Climas. En: González-Soriano E., Dirzo R. y Vogt R. Eds. Historia Natural de Los Tuxtlas, pp. 7-23, Universidad Nacional Autónoma de México, México, D.F.

Vázquez-Yanes C. y Orozco-Segovia A. 1990. Ecology of light controlled seed germination in two contrasting tropical habitats. Oecología 83:171-175.

Venable L. 1988. The selective interactions of dispersal, dormancy and seed size as adaptations for reducing risk in variable environment. American Naturalist 3:360-384.

Westoby M., Jurado E. y Leishman M. 1992. Comparative evolutionary ecology of seed size. Trends in Ecology and Evolution 7:368-372.

Whitmore T. 1978. Gaps in the forest canopy. En: Tomlinson P. y Zimmerman M. Eds. Tropical Trees as Living Systems, pp. 655-669, Cambridge, Londres.

Zar J.H. 1999. Biostatistical Analysis. Prentice Hall, Upper Saddle River.

Fecha de recepción: 30 de agosto 2002

Versión corregida: 2 de marzo de 2004

Aceptado: 3 de marzo de 2004 